\title{
Multichannel Inversion of Scattered Teleseismic Body Waves: Practical Considerations and Applicability
}

\author{
Stéphane Rondenay \\ Department of Earth, Atmospheric and Planetary Sciences, Massachusetts Institute of Technology, Cambridge, Massachusetts \\ Michael G. Bostock \\ Department of Earth and Ocean Sciences, University of British Columbia, Vancouver, Canada
}

Karen M. Fischer

Department of Geological Sciences, Brown University, Providence, Rhode Island

\begin{abstract}
We investigate the resolving power and applicability of a recently developed technique for multichannel inversion of scattered teleseismic body waves recorded at dense seismic arrays. The problem is posed for forward- and back-scattered wavefields generated at discontinuities in a 2D isotropic medium, with the backprojection operator cast as a generalized Radon transform (GRT). The approach allows for the treatment of incident plane waves from arbitrary backazimuths, and recovers estimates of material property perturbations about a smoothly varying reference model. An investigation of the main factors affecting resolution indicates that: (1) comprehensive source/station coverage is necessary to optimize geometrical resolution and recover accurate material property perturbations; (2) the range in dip resolution diminishes with increasing depth and is inversely proportional to array width (e.g., reaches $\left[-45^{\circ}, 45^{\circ}\right]$ at depths equivalent to $\sim 1 / 2$ array width); (3) distortion of the image due to spatial aliasing is only significant at depths $\leq 2$ $\times$ [station spacing]; and (4) unaccounted for departures from model assumptions (i.e., isotropy and 2D geometry) result in defocusing and mismapping of structure. Two applications to field data are presented. The first considers data from the Abitibi 1996 broadband array, in which stations were deployed at $20 \mathrm{~km}$ intervals. Imaging results show that this level of spatial sampling, which is characteristic of modern broadband arrays, is sufficient to adequately resolve structure below mid-crustal depths. For these data, we introduce a new preprocessing algorithm that uses eigenimage decomposition of seismic sections to suppress wavefield contamination by $P c P$ and $P P$ phases. The second application involves short period data from the Los Angeles Region Seismic Experiment and shows that images obtained from high frequency records are subject to significant contamination by scattered surface waves.
\end{abstract}

Seismic Earth: Array Analysis of Broadband Seismograms

Geophysical Monograph Series 157

Copyright 2005 by the American Geophysical Union

10.1029/157GM12 


\section{INTRODUCTION}

One of the current challenges faced by earthquake seismologists is the development of new tools for high-resolution imaging of the lithosphere and underlying mantle. While tomographic methods using transmitted body waves [Aki et al., 1977; VanDecar, 1991; Grand, 1994; van der Hilst et al., 1997] and surface waves [van der Lee and Nolet, 1997; Li et al., 2002] have proven to be powerful tools for volumetric imaging of seismic anomalies, a wealth of complementary information on internal discontinuities can be obtained from secondary scattered waves in the coda of body waves. The relative arrival time and amplitude of these secondary waves are indicative of the position and magnitude of discontinuities in elastic parameters of the subsurface. The teleseismic $P$ wavefield is of particular interest as it benefits from generally higher signal-to-noise ratio than other (later arriving) primary phases. Scattered waves, notably $P$-to- $S$ conversions, recorded at single stations have thus been utilized for over two decades as a basis for 1-D modelling of planar discontinuities in the lithosphere [Vinnik, 1977; Langston, 1979]. In recent years, a substantial increase in availability and fidelity of recording instruments has led to new opportunities for multichannel processing of scattered teleseismic body waves, allowing for 2D/3D imaging of subsurface structure. Many of these approaches are similar to industryoriented (e.g., migration) techniques and generate analogous high-resolution depth sections of the subsurface, down to the mantle transition zone.

Recently developed methods form a continuum with respect to the level of complexity adopted in the treatment of the scattering problem. On one end of the spectrum, images may be produced by simple stacking of normalized $P$-to- $S$ conversion records (i.e., receiver functions - RF), which are binned according to common conversion points (CCP) and mapped to depth [Dueker and Sheehan, 1997]. Finer resolution can be achieved through the stacking of singly scattered wavefields along diffraction hyperbolae to recover relative scattering intensity/potential at individual points through a 2D or 3D model space [Revenaugh, 1995a; Ryberg and Weber, 2000; Sheehan et al., 2000]. Moving to higher levels of complexity, methods involving inversion/backprojection operators [Bostock and Rondenay, 1999a-b; Bostock et al., 2001; Poppeliers and Pavlis, 2003a-b] and full 3D waveform inversion [Frederiksen and Revenaugh, 2002] of scattered teleseismic body waves recover either the scattering potential or estimates of localized material property perturbations with respect to an a priori background model.

In this paper, we explore the domains of application of a 2D inversion/backprojection approach in which the problem is cast as a generalized Radon transform (GRT) [Bostock et al., 2001]. Throughout this text, the method will be referred to as "2D GRT inversion". To this day, it has been tested on synthetic data [Shragge et al., 2001] and has been successfully applied to field data from the Cascadia 1993-94 (CASC93) experiment [Rondenay et al., 2001]. Factors that made CASC93 an ideal data set for this exercise were a combination of very high station density $(\sim 5 \mathrm{~km}$ station spacing) and signal quality. In anticipation of further improvements in sampling density of three-component broadband seismometers [e.g., USARRAY, Levander et al., 1999] we undertake an assessment of the validity of the method in a variety of field contexts. Our main objective is thus to provide a set of guidelines for applications of the method and identify avenues for possible future improvements. Of particular interest is the performance of the technique in imaging subsurface structure using datasets recorded under less ideal conditions than CASC93.

We begin this study with a short summary of the theoretical framework for 2D GRT inversion and a description of the preprocessing steps necessary to render the data amenable to analysis. This is followed by a discussion of the theoretical and practical factors that affect the resolving power of the method. We devise a set of guidelines to determine the expected spatial resolution afforded by the technique as a function of signal bandwidth, array aperture and station spacing. We then investigate the behaviour of 2D GRT inversion when it is applied to field data sets that do not strictly meet its geometrical requirements. We present examples from two Incorporated Research Institutions for SeismologyProgram for Array Seismic Studies of the Crust and Lithosphere (IRIS-PASSCAL) deployments: Abitibi 1996 (ABI96) and the 1993 Los Angeles Region Seismic Experiment (LARSE). The study concludes with a discussion of the key results and future improvements that may further refine the resolution of subsurface structure.

\section{INVERSION METHOD AND DATA PREPARATION}

\subsection{Background and Theory}

The theoretical framework for the 2D GRT inversion is based on original work by Beylkin [1985; see also Beylkin and Burridge, 1990; Miller et al., 1987] for controlled source data. The problem was cast for teleseismic wavefields by Bostock et al. [2001], as an extension of the teleseismic migration approach suggested by Bostock and Rondenay [1999a-b]. Both of these studies consider the interaction of an incident, planar teleseismic wavefield with 2D lithospheric structure and the generation of resultant scattered waves. Lithospheric structure is defined here as a local 2D perturbation in isotropic material properties, for example $P$-wave 
velocity $\left(\Delta \alpha / \alpha_{0}\right), S$-wave velocity $\left(\Delta \beta / \beta_{0}\right)$ or density $\left(\Delta \rho / \rho_{0}\right)$ on a $1 \mathrm{D}$ reference medium $\left(\alpha_{0}, \beta_{0}, \rho_{0}\right)$. The total wavefield, comprising both incident and scattered waves, is recorded on a dense, quasi-linear array of receivers situated on the Earth's surface. Two key aspects of the method outlined in Bostock et al. [2001] serve to render it generally applicable to field data sets: first, it accommodates incident wavefields arriving from arbitrary back azimuths; second, it allows for the analysis of both forward- and back-scattered waves, the latter being afforded by free-surface reflection/conversion of the incident wave.

The 2D GRT inversion is based on a high-frequency (i.e., ray-theoretical), single-scattering formulation of the forward problem. In this approach, the scattered displacement field for a particular scattering mode interaction (either forward or back scattered) recorded at the surface and resulting from a planar incident wave can be expressed as [simplified from Bostock et al., 2001]:

$$
\begin{aligned}
& \boldsymbol{\Delta} \boldsymbol{u}\left(\mathbf{x}^{\prime}, \mathbf{p}_{\perp}^{0}, t\right)= \int \mathrm{d} \mathbf{x} \mathcal{V}\left(\mathbf{x}, \mathbf{x}^{\prime}, \mathbf{p}_{\perp}^{0}\right) f(\mathbf{x}, \boldsymbol{\theta}) \\
& \cdot \delta\left[t-\mathcal{T}\left(\mathbf{x}, \mathbf{x}^{\prime}, \mathbf{p}_{\perp}^{0}\right)\right],
\end{aligned}
$$

where vector quantities are indicated in boldface. 2D spatial variables $\mathbf{x}, \mathbf{x}^{\prime}$ represent line-scatterer and line-receiver locations, respectively, and lie within the $x_{1}, x_{3}$ plane (i.e., $2 \mathrm{D}$ strike parallels $x_{2}$-coordinate, with $x_{1}$ orthogonal in horizontal plane and $x_{3}$ vertical, positive downward); and $\mathbf{p}_{\perp}^{0}=$ $\left(p_{1}^{0}, p_{2}\right)$ is the horizontal slowness of the incident wave. The scattering potential,

$$
f(\mathbf{x}, \theta)=\sum_{l=1}^{3} W_{l}(\theta) \Delta m_{l}(\mathbf{x}),
$$

includes radiation pattern coefficients $W_{l}(\theta)$, which are dependent on the scattering angle $\theta$ between the incident and scattered waves, and the material property perturbations $\Delta m_{l}=\left(\Delta \alpha / \alpha_{0}, \Delta \beta / \beta_{0}, \Delta \rho / \rho_{0}\right)$. Quantity $\mathcal{T}\left(\mathbf{x}, \mathbf{x}^{\prime}, \mathbf{p}_{\perp}^{0}\right)$ is the arrival time at receiver $\mathbf{x}^{\prime}$ of energy scattered from $\mathbf{x}$, and $\mathcal{V}\left(\mathbf{x}, \mathbf{x}^{\prime}, \mathbf{p}_{\perp}^{0}\right)$ combines a series of filters and weights that include geometrical amplitudes of the incident and scattered waves.

The forward problem, as expressed in Equation (1), is represented schematically in Figure 1a for $P$-to- $S$ transmission scattering. The scattered displacement observed at a given time $t$ and receiver position $\mathbf{x}^{\prime}$ in data space corresponds to the sum of contributions from all scatterers $\mathbf{x}$ in model space that can generate signal at that data point. These scatterers are distributed along an isochronal surface (i.e., $t=\mathcal{T}\left(\mathbf{x}, \mathbf{x}^{\prime}, \mathbf{p}_{\perp}^{0}\right)$ constant) which is defined by the ray kinematics in the reference background model. Transmission scatter-

\section{a) FORWARD PROBLEM}
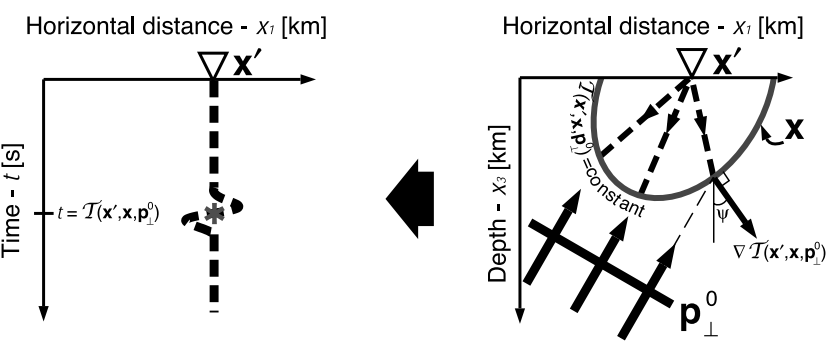

b)
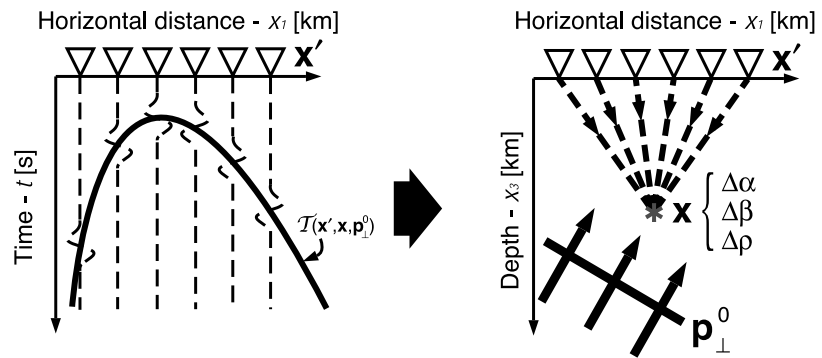

Figure 1. Schematic representation of forward and inverse scattering problems for $P$-to- $S$ scattering in terms of model space (i.e., scatterer location) and data space (i.e., station position versus time). a) Forward problem expresses the scattered displacement field measured at a given time $t=\mathcal{T}\left(\mathbf{x}, \mathbf{x}^{\prime}, \mathbf{p}_{\perp}^{0}\right)$ and station location $\mathbf{x}^{\prime}$, due to all point scatterers in model space that could contribute energy to that point in data space. b) Inverse problem involves weighted diffraction stack along the traveltime curves corresponding to a point scatterer at $\mathbf{x}$ and recovers material property perturbations $\Delta m_{l}=\left(\Delta \alpha / \alpha_{0}, \Delta \beta / \beta_{0}, \Delta \rho / \rho_{0}\right)$ at that point (shown here for a single source).

ing is characterized by an isochron describing a constant travel-time delay between the upgoing incident wave and the scattered wave. Additional treatment of back-scattered waves is afforded by calculating the isochrons corresponding to the downgoing incident wavefield, i.e. that portion of the incident wavefield which has been reflected from the freesurface before interacting with a scatterer [see Bostock et al., 2001; Rondenay et al., 2001].

As originally recognized by Beylkin [1985], the scattered displacement field as expressed in equation (1) possesses a form which closely resembles the Radon transform in that it involves an integration over surfaces (or lines in 2D). This analogy with the Radon transform (and its formal inverse) provides the basis for the development of a back-projection operator to the scattering problem. The 2D Radon transform pair is given by

$$
F(\mathbf{n}, s)=\int \mathrm{d} \mathbf{x} f(\mathbf{x}) \delta(s-\mathbf{n} \cdot \mathbf{x}),
$$




$$
\begin{aligned}
f\left(\mathbf{x}_{0}\right) & =-\frac{1}{4 \pi} \int \mathrm{d} \mathbf{n} \mathcal{H}\left[\left.\frac{\partial}{\partial s} F(\mathbf{n}, s)\right|_{s=\mathbf{n} \cdot \mathbf{x}_{0}}\right] \\
& =-\frac{1}{4 \pi} \int \mathrm{d} \mathbf{n} \int \mathrm{d} \mathbf{x} f(\mathbf{x}) \mathcal{H}\left\{\delta^{\prime}\left[\mathbf{n} \cdot\left(\mathbf{x}_{0}-\mathbf{x}\right)\right]\right\} \\
& =-\frac{1}{4 \pi} \int_{0}^{2 \pi} \mathrm{d} \psi \int \mathrm{d} \mathbf{x} f(\mathbf{x}) \mathcal{H}\left\{\delta^{\prime}\left[\mathbf{n} \cdot\left(\mathbf{x}-\mathbf{x}_{0}\right)\right]\right\}
\end{aligned}
$$

where the surfaces of integration in (3) are planes defined by their normals $\mathbf{n}$ and their distances from the origin $s$, angle $\psi$ defines the direction of $\mathbf{n}$, and $\mathcal{H}\{\cdot\}$ denotes the Hilbert transform. The definition of the inverse Radon transform in (4) indicates that it is possible to reconstruct a function $f(\mathbf{x})$ at any point $\mathbf{x}_{0}$ by summing $F(\mathbf{n}, s)$ over all planes that pass through this point. Unlike the Radon transform, the isochronal surfaces of integration in (1) are curved (see e.g., Figure 1a), but can be approximated as planes when evaluating the integral in the vicinity of a point $\mathbf{x}_{0}$. A back-projection operator for the scattering problem can be constructed by identifying $\boldsymbol{\Delta} \boldsymbol{u}\left(\mathbf{x}^{\prime}, \mathbf{p}_{\perp}^{0}, t\right)$ with $F(\mathbf{n}, s)$ in (4), with $\mathbf{n}$ and $s$ corresponding to traveltime gradient $\nabla \mathcal{T}$ and traveltime $t$, respectively [see Miller et al., 1987]. This operator has the form:

$$
\begin{gathered}
f\left(\mathbf{x}_{0}, \theta\right)=\frac{1}{4 \pi} \int \mathrm{d} \psi \mathrm{y}\left(\mathbf{x}_{0}, \mathbf{x}^{\prime}, \mathbf{p}_{\perp}^{0}\right) \\
\cdot \Delta \boldsymbol{u}\left[\mathbf{x}^{\prime}, \mathbf{p}_{\perp}^{0}, t=\mathcal{T}\left(\mathbf{x}_{0}, \mathbf{x}^{\prime}, \mathbf{p}_{\perp}^{0}\right)\right],
\end{gathered}
$$

where all weights and filters applied to the scattered displacement, including those introduced to replicate the Radon transform, are grouped under the term $\mathcal{Y}\left(\mathbf{x}_{0}, \mathbf{x}^{\prime}, \mathbf{p}_{\perp}^{0}\right)$. Note that, within the assumption of high-frequency single scattering, the operator in (5) can reconstruct exactly the function $f\left(\mathbf{x}_{0}, \theta\right)$ only if the coverage in $\psi$ is continuous and complete over $[-\pi / 2, \pi / 2]$. Since this requirement is never met in realistic field conditions, the resolving power of the operator will be degraded as discussed in section 3 .

Using equation (5) and the definition of the scattering potential $f\left(\mathbf{x}_{0}, \theta\right)$ in (2), the material property perturbations at any scatter point in the model $\Delta \mathrm{m}\left(\mathbf{x}_{0}\right)=$ $\left[\Delta \alpha / \alpha_{0}, \Delta \beta / \beta_{0}, \Delta \rho / \rho_{0}\right]^{\mathrm{T}}$ can be obtained by solving a small linear system, leading to the expression

$$
\Delta \mathrm{m}=\mathrm{H}^{-1} \mathrm{~g} .
$$

Note that this system can be solved simultaneously for all scattering modes (i.e., forward- and back-scattering) and incident wavefields represented within a data set. The elements of $g$ are given by

$$
\begin{gathered}
g_{1}\left(\mathbf{x}_{0}\right)=\frac{1}{4 \pi} \int \mathrm{d}\left|\mathbf{p}_{\perp}^{0}\right| \int \mathrm{d} \gamma \int \mathrm{d} x^{\prime}{ }_{1}\left|\frac{\partial(\psi, \theta)}{\partial\left(x^{\prime}, \gamma\right)}\right| W_{l}(\theta) \\
\cdot y\left(\mathbf{x}_{0}, \mathbf{x}^{\prime}, \mathbf{p}_{\perp}^{0}\right) \cdot \boldsymbol{\Delta} \boldsymbol{u}\left[\mathbf{x}^{\prime}, \mathbf{p}_{\perp}^{0}, t=\mathcal{T}\left(\mathbf{x}_{0}, \mathbf{x}^{\prime}, \mathbf{p}_{\perp}^{0}\right)\right]
\end{gathered}
$$

where $\gamma$ is the event backazimuth, such that $\tan (\gamma)=$ $p_{2} / p_{1}^{0}$. The determinant in the integrand of equation (7) is a Jacobian (or "Beylkin determinant") that permits practical integration over variables representing source $\left(\left|\mathbf{p}_{\perp}^{0}\right|, \gamma\right)$ and receiver position $\mathbf{x}^{\prime}=\left(x_{1}^{\prime}, 0\right)$. The elements of the Hessian matrix $\mathrm{H}$ are given by

$$
H_{l k}\left(\mathbf{x}_{0}\right)=\int_{\cdot W_{k}\left(\theta,\left|\mathbf{p}_{\perp}^{0}\right|, \mathbf{x}_{0}\right)} \mathrm{d}\left|\mathbf{p}_{\perp}^{0}\right| \int_{W_{\perp}} \mathrm{d} \theta W_{l}\left(\theta,\left|\mathbf{p}_{\perp}^{0}\right|, \mathbf{x}_{0}\right)
$$

The inverse problem can, according to equations (7-8), be viewed simply as a weighted diffraction stack over all sources and receivers (with weights determined by the analogy with the Radon transform), followed by a trivial $3 \times 3$ matrix inversion. A schematic representation of the inverse problem is displayed in Figure $1 \mathrm{~b}$ for a single incident wavefield. The inverse procedure is repeated for all image points in model space to obtain a depth section of velocity and density perturbations.

\subsection{Data Preprocessing}

Prior to inversion, the raw data must be preprocessed to effectively remove the incident wavefield, thereby isolating the scattered displacement field (i.e., $\left.\Delta \boldsymbol{u}\left(\mathbf{x}^{\prime}, \mathbf{p}_{\perp}^{0}, t\right)\right)$. This preprocessing involves several steps which are presented in detail by Bostock and Rondenay [1999a], and can be summarized as follows (see Figure 2):

(1) Transform displacement $\mathrm{u}=\left[u_{R}, u_{T}, u_{Z}\right]^{T}$ (where subscripts $\mathrm{R}, \mathrm{T}$ and $\mathrm{Z}$ correspond to radial, transverse and vertical directions, respectively) to an upgoing wave-vector space $\mathrm{w}=[P, S C 1, S C 2]^{T}$ using the inverse free-surface transfer matrix [Kennett, 1991]. This transformation yields a robust estimate of the incident $P$-wavefield, which is isolated in a single trace denoted $P$. Traces $S C 1$ and $S C 2$ are estimates of the $S V$ and $S H$ components of the incident wavefield, respectively, and should therefore contain only scattered energy.

(2) Time-normalize (i.e. align) the resulting $P$-wave section using multi-channel cross-correlation derived delay times [VanDecar and Crosson, 1990].

(3) Estimate and separate incident (I) and scattered (SC3) wavefields by principal component (i.e., eigenimage) decomposition of the $P$-wave section [Ulrych et al., 1998]. I is obtained by reconstructing the section using only the 


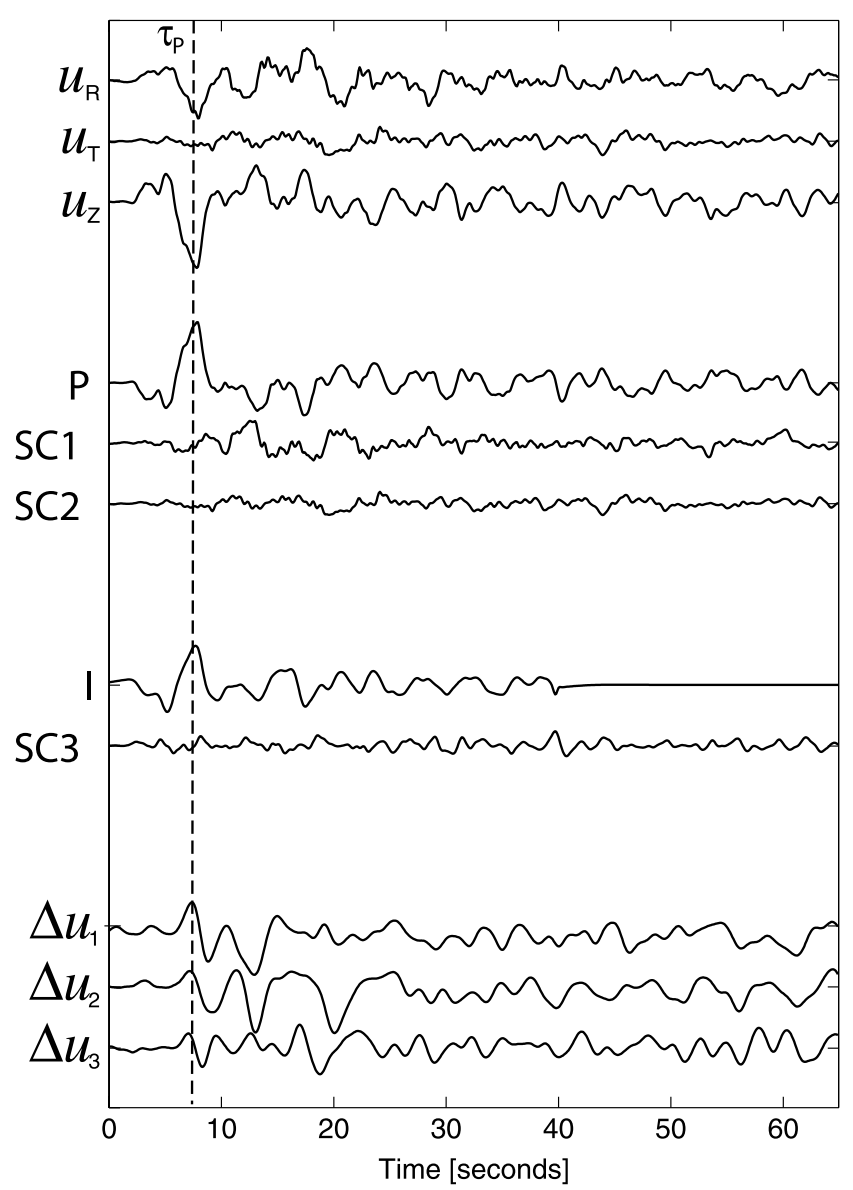

Figure 2. Preprocessing of the $P$-coda from a Mexico earthquake (Sept. 3, 1993; 12:35:00 UT; $14.52^{\circ} \mathrm{N},-92.71^{\circ} \mathrm{E}, 27 \mathrm{~km} ; m_{\mathrm{b}}=$ 5.8) recorded at station $\mathrm{A} 03\left(44.42^{\circ} \mathrm{N},-123.95^{\circ} \mathrm{E}\right)$ of the CASC93 deployment. Seismic traces represent, from top to bottom: radial, transverse and vertical components of the raw data $\left(u_{R}, u_{T}, u_{Z}\right)$; decomposed $P$-, SC1 - and SC2- components; estimates of the incident $(I)$ and scattered (SC3) wavefields determined from principal component analysis; scattered displacement field in the $x_{1}, x_{2}$ and $x_{3}$ directions, bandpass filtered between $0.03-0.3 \mathrm{~Hz}\left(\Delta u_{1}, \Delta u_{2}, \Delta u_{3}\right)$.

first or first few principal components, which represent that portion of the signal most strongly correlated from trace to trace. $S C 3$ is assembled using the remaining principal components, as an estimate of the scattering contribution to the $P$-wave section.

(4) Reconstitute the upgoing scattered wavefield $\Delta \mathrm{u}_{\mathrm{RTZ}}=\left[\Delta u_{R}, \Delta u_{T}, \Delta u_{Z}\right]^{T}$ from $\mathrm{w}^{\prime}=[S C 3, S C 1$, $S C 2]^{T}$ using the eigenvector matrix for 1D media [e.g., Bostock and Rondenay, 1999a].

(5) Deconvolve the estimated source-time function $I$ from the reconstituted displacement sections $\Delta \mathrm{u}_{\mathrm{RTZ}}$ to obtain the scattered wave impulse response. The deconvolution is performed by spectral division, with regularization determined through minimization of oscillations in the resulting impulse response.

These first five steps lead to estimates of the scattered components of the Green's function $\boldsymbol{\Delta} \boldsymbol{u}\left(\mathbf{x}^{\prime}, \mathbf{p}_{\perp}^{0}, t\right)$. Two additional steps are required to render the data sections amenable to 2D GRT inversion:

(6) $2 \mathrm{D}$ treatment requires rotation of horizontal displacement into a reference frame aligned with the inferred tectonic strike of the study area (i.e., $x_{2}$ parallel to $2 \mathrm{D}$ regional strike). (7) Convolve the resulting data sections with the filter $i \operatorname{sgn}(\omega) / \sqrt{-i \omega}$, which is included in term $\mathcal{Y}\left(\mathbf{x}_{0}, \mathbf{x}^{\prime}, \mathbf{p}_{\perp}^{0}\right)$ of equations (5) and (7), and is required through 2D GRT to recover material property perturbations $\Delta \mathrm{m}$ [see Bostock et al., 2001, their equation (29)].

\section{RESOLUTION}

The efficacy of 2D GRT inversion for the recovery of subsurface structure depends on the combined effects of array and source configuration. As discussed in Bostock et al. [2001], resolution is a measure of the accuracy in recovering both the geometry (depth, volume and dip) of discontinuities and the amplitude of associated material property perturbations. In this section, we consider several aspects of the theoretical framework and applications to synthetic and field data to draw a set of basic observations and guidelines regarding resolution.

\subsection{Aperture-Completeness of Coverage}

The analogy between forward/inverse scattering problems and the Radon Transform is the fundamental basis of 2D GRT inversion. As discussed in detail by Bostock et al. [2001], the validity of this analogy relies in large part on the coverage in total traveltime gradient $\nabla \mathcal{T}$ at each image point (see Figure 1). Specifically, the magnitude $|\nabla \mathcal{T}|$ represents the sensitivity of traveltime to scatterer location, whereas its orientation $\psi$ controls dip resolution, such that structure dipping perpendicularly to sampling in $\nabla \mathcal{T}$ will be well resolved. Volume resolution is controlled by an effective wave-number $\omega|\nabla \mathcal{T}|$, where $\omega$ represents the angular frequency of the scattered signal [Bostock et al., 2001]. Therefore, a high degree of 2D spatial resolution will be insured by large values of $|\nabla \mathcal{T}|$, a large signal bandwidth, and complete directional coverage in $\nabla \mathcal{T}$ (i.e., $\psi=[-\pi /$ $2, \pi / 2]$ ). In practice, these conditions are only partially met for realistic field conditions. As a result, geometrical resolution is reduced, causing distortion in the image. Moreover, the recovery of accurate amplitudes of material property perturbations can be greatly reduced, if not completely lost, if coverage is inadequate. 
Aperture and coverage issues are illustrated in Figure 3, which shows the sampling in $\nabla \mathcal{T}$ achieved in an experimental setup for $P-S$ back-scattering at a single image point. The top panels represent sampling in $\nabla \mathcal{T}$ afforded by an idealized station coverage, whereas the lower panels panels show sampling at an image point beneath the ABI96 array (see section 4.1). In the idealized case, the coverage in $\psi$ is nearly uniform over the range $\left[-70^{\circ}, 70^{\circ}\right]$, with all directions sampled by values of $\nabla \mathcal{T}>0.32 \mathrm{~s} / \mathrm{km}$. This is equivalent to the coverage that was afforded by the CASC93 data set [Rondenay et al.,2001], which produced realistic $S$-velocity perturbations of $\pm 5 \%$ [see, also, Bostock et al., 2002]. In contrast, the coverage afforded by the ABI96 data is much poorer in both $\psi$ and $|\nabla \mathcal{T}|$, with considerable gaps present in the $\psi=\left[-20^{\circ}, 20^{\circ}\right]$ interval. The resulting loss of resolution and inability to recover accurate property perturbations is discussed in section 4.1. This type of analysis is useful to assess the resolving power of 2D GRT inversion in different portions of model space as a function of station and source coverage.

Lastly, we observe that for a fixed array width and complete range of teleseismic incidences, the resolution tends to decrease with depth as the coverage in $\nabla \mathcal{T}$, and especially the range in $\psi$, tend to diminish. Recalling the idealized example in Figure $3 \mathrm{a}-\mathrm{b}$ with an array width of $500 \mathrm{~km}$, the range in $\psi$ is reduced from $\left[-70^{\circ}, 70^{\circ}\right]$ at $40 \mathrm{~km}$ depth to $\left[-60^{\circ}, 60^{\circ}\right]$ at $100 \mathrm{~km}$ and $\left[-33^{\circ}, 33^{\circ}\right]$ at $500 \mathrm{~km}$. This implies that at $500 \mathrm{~km}$ depth, structure with dips greater than $33^{\circ}$ will not be resolved. Provided that the source distribution is complete and the sampling density is adequate, the main factor controlling the geometrical resolution with increasing depth is array width. It is thus possible to determine a maximum depth of resolution by choosing a minimum range in $\psi$ coverage for the center of the array. For example, with an average global background velocity model, a range of $\left[-45^{\circ}, 45^{\circ}\right]$ will be reached at a depth equivalent to approximately half the array width. Note, however, that moving the image point toward the edge of the model tends to reduce the $\psi$ range and shift its mean value. Accordingly, at a depth corresponding to half the array width, we find that $\psi \sim\left[-60^{\circ}, 15^{\circ}\right]$ on the left [i.e., $\left.\min \left(x_{1}\right)\right]$ side of the model and $\psi \sim\left[15^{\circ},-60^{\circ}\right]$ on the right side [i.e., $\left.\max \left(x_{1}\right)\right]$.

\subsection{Spatial Aliasing}

The surface density of recording instruments strongly affects the resolving power of 2D GRT inversion, as it controls spatial sampling of the scattered signal. Indeed, as extensively documented by the active source community [e.g., Yilmaz, 1987], a degradation in geometrical resolution arises when applying imaging/migration operators to signal that is spatially undersampled (i.e., aliased). Below, we discuss two forms of aliasing that affect images created by 2D GRT inversion: spatial aliasing and operator aliasing.

Spatial aliasing will occur when station density at the surface is such that the incoming scattered wavefield is sampled at intervals greater than half its apparent wavelength $\lambda$. For a 1D layered model, the maximum station separation $\Delta x_{1}$ to avoid spatial aliasing can be calculated by considering the following two equations:

$$
\begin{aligned}
& \Delta x_{1}=\mathrm{x}_{1}^{\prime \prime}-x_{1}^{\prime}=\int_{0}^{z} \mathrm{~d} x_{3}\left(\frac{p_{1}^{\prime \prime}}{\eta^{\prime \prime}\left(x_{3}\right)}-\frac{p_{1}^{\prime}}{\eta^{\prime}\left(x_{3}\right)}\right), \\
& \Delta n \lambda=\int_{0}^{z} \frac{\mathrm{d} x_{3}}{v^{2}\left(x_{3}\right) T}\left(\frac{1}{\eta^{\prime \prime}\left(x_{3}\right)}-\frac{1}{\eta^{\prime}\left(x_{3}\right)}\right) \leq 0.5,
\end{aligned}
$$

where $\Delta n \lambda$ is the cumulative difference in wavelength between two rays scattered from a same subsurface location $\mathbf{x}_{0}=(0, z)$ to receivers $\mathbf{x}^{\prime}=\left(x_{1}^{\prime}, 0\right)$ and $\mathbf{x}^{\prime \prime}=\left(x_{1}^{\prime \prime}, 0\right) ; v\left(x_{3}\right)$ is the wavespeed of the scattered rays; $T$ is the dominant period of the scattered wavefield; $p_{1}^{\prime}$ and $p_{1}^{\prime \prime}$ are the horizontal slowness components of the two scattered rays; $\eta^{\prime}\left(x_{3}\right)$ and $\eta^{\prime \prime}\left(x_{3}\right)$ are the vertical slowness components (i.e., $\eta^{\prime}\left(x_{3}\right)=$ $\sqrt{1 / v^{2}\left(x_{3}\right)-\left(p_{1}^{\prime}\right)^{2}-p_{2}^{2}}$, with $p_{2}$ as that of incident wave). Axes are defined as in the right panels of Figure 1. For a given receiver location $x_{1}^{\prime}$, the maximum spacing $\Delta x_{1}$ is obtained from (9) for the minimum value of $\eta^{\prime \prime}\left(x_{3}\right)$ that satisfies (10).

In the simple case of a plane wave propagating through a homogeneous half-space, the above system reduces to:

$$
\Delta x_{1}=\frac{z}{\eta^{\prime}}\left[\frac{\left(0.5 v^{2} T \eta^{\prime}+z\right)^{2}-\left(z \eta^{\prime} v\right)^{2}}{v z}-p_{1}^{\prime}\right]
$$

for a given receiver location $x_{1}^{\prime}$, and constant scattered wavespeed $v$. From (11), it can be shown that the station spacing required to avoid spatial aliasing approaches asymptotically $\lambda / 2$ (within $10 \%$ for incidence angles $\gtrsim 65^{\circ}$ ), a situation that is generally encountered for $x_{1}^{\prime}>2 z$. Assuming an average crustal $S$-velocity of $3.6 \mathrm{~km} / \mathrm{s}$, the station spacing must therefore be no larger than $1.8-18 \mathrm{~km}$ to adequately sample signal with dominant frequencies ranging over the interval 1-0.1 Hz. Spatial aliasing considered in these terms will thus affect the images obtained with 2D GRT inversion if the station density is too sparse. However, the negative effects of aliasing will be offset, in part, by the broad signal bandwidth of the teleseismic $P$-wavefield and the simultaneous processing of multiple incident waves and scattering modes.

Operator aliasing occurs when the migration stacking operator does not sample the data adequately. This type of 


\section{Idealized aperture}
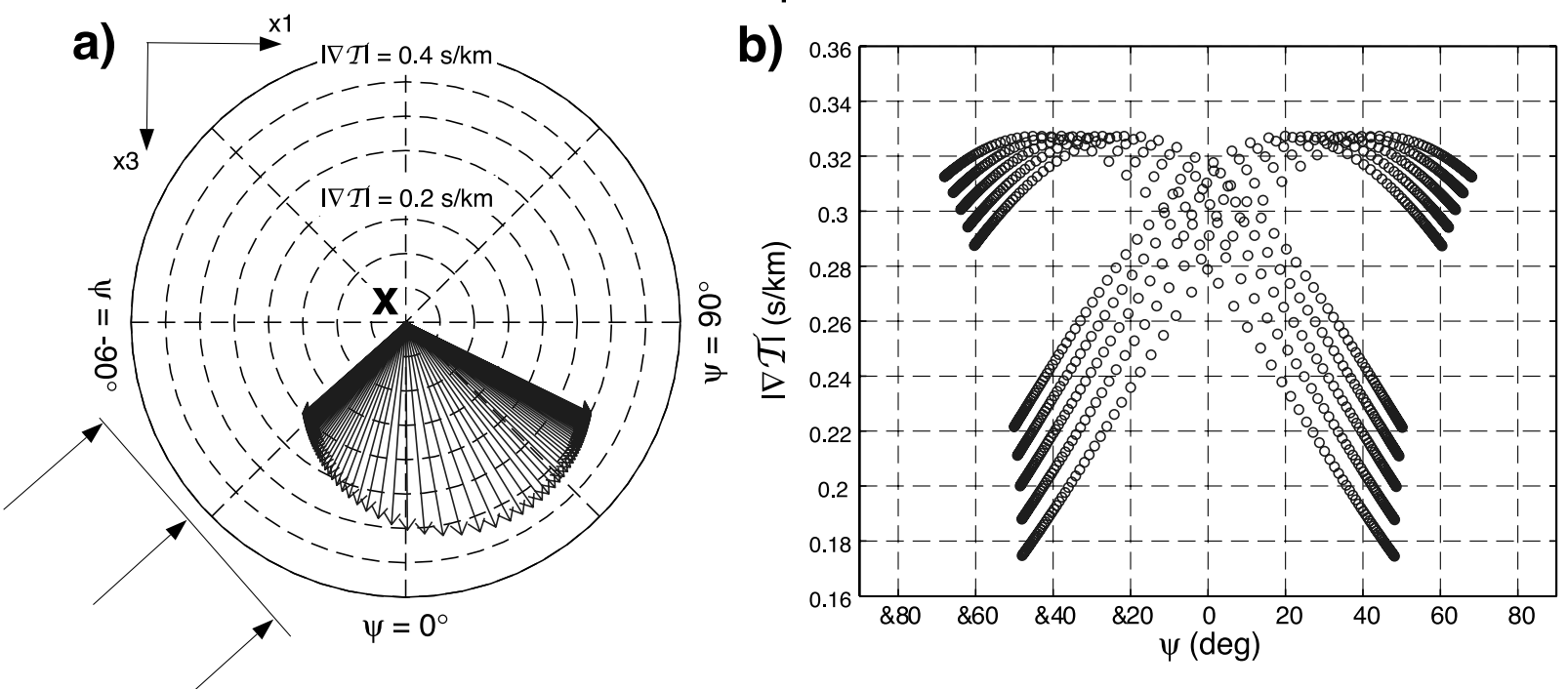

ABI96 experiment
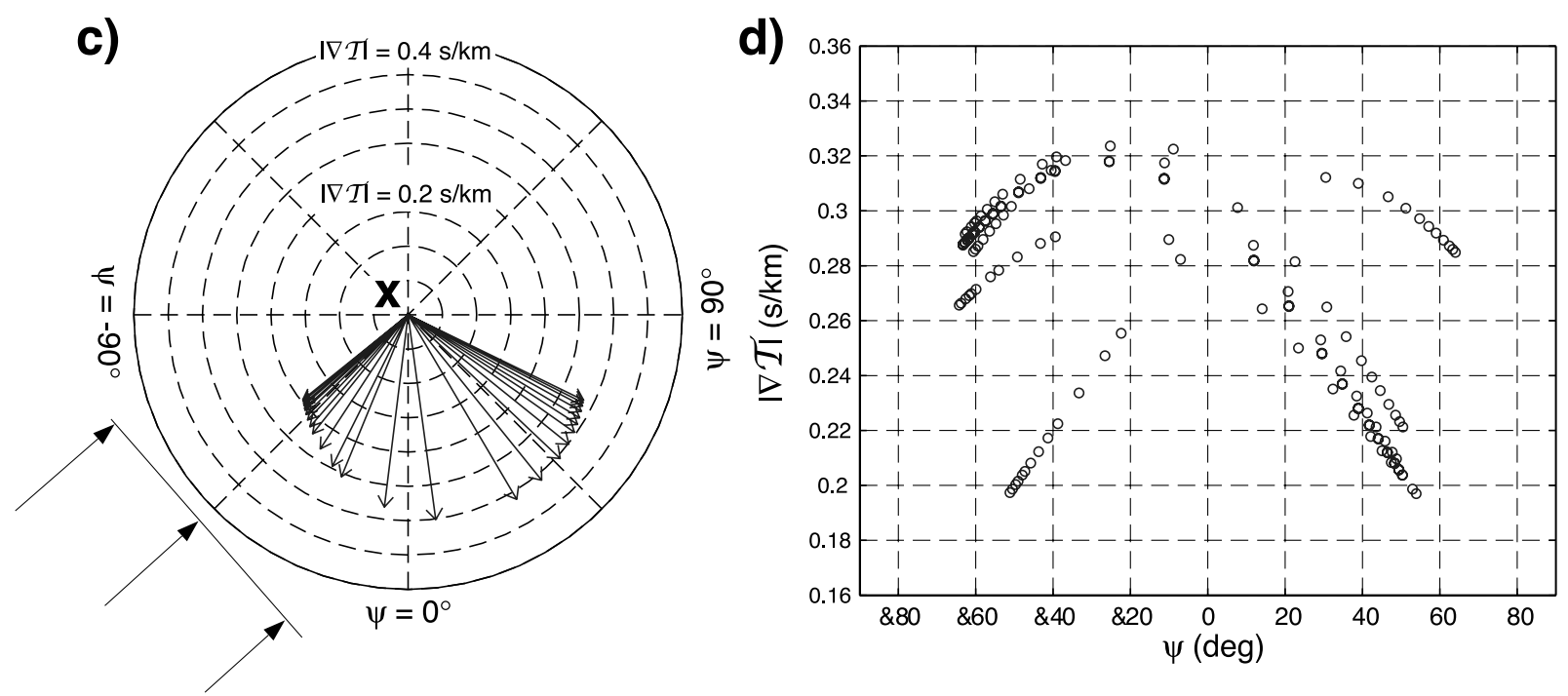

Figure 3. Aperture (i.e., coverage in $\nabla \mathcal{T}$ ) as a function of station and source distribution, for a single image point located at $40 \mathrm{~km}$ depth beneath the center of a $500 \mathrm{~km}$-long array. Top panels: idealized scenario with $5 \mathrm{~km}$ station spacing for (a) a single plane wave incident from the left on image point $\mathbf{x}$ (see Figure 1 for description of $x_{1}-x_{3}$ axes), and (b) incident waves at a full range of teleseismic distances (i.e., $\sim 30-100^{\circ}$ ). Lower panels: image point beneath the ABI96 array (see section 4.1) with irregular station spacing of $\sim 15-25 \mathrm{~km}$ for (a) a single plane wave incident from the left, and (b) the complete ray coverage afforded by the data set. Note that for single incident waves (i.e., left panels), the coverage in $\nabla \mathcal{T}$ is represented as vectors centered on the image point, whereas for complete ray coverage (i.e., right panels) the values are plotted graphically. 
aliasing has been documented in the seismic reflection literature, where low-pass filtering and wavenumber-dependent filtering based on a priori knowledge of the data dip spectrum have been proposed as counteracting antialiasing operators [see, e.g., Gray, 1992; Biondi, 2001]. In the specific case of 2D GRT inversion, operator aliasing is related to undersampling of GRT-derived stacking weights (i.e., $\frac{1}{4 \pi}\left|\frac{\partial(\psi, \theta)}{\partial\left(x^{\prime}, \gamma\right)}\right| W_{l}(\theta) y\left(\mathbf{x}_{0}, \mathbf{x}^{\prime}, \mathbf{p}_{\perp}^{0}\right)$ in equation 7). Applications to sparsely sampled synthetic data [Shragge et $a l ., 2001]$ and field data (section 4.1) show that operator aliasing is manifest through a high wave number "speckle" in the resulting image. This effect tends however to be predominant near the surface and diminish with increasing depth. It is related to rapid variations in traveltime sensitivity $\nabla \mathcal{T}$ near the surface, which produce sharp peaks in the total weighting function of the diffraction hyperbola. A high spatial density of data in thus necessary to adequately sample these peaks. As depth increases, however, the weighting function becomes smoother and can be representatively sampled by sparser recording points. This form of aliasing is illustrated in Figure 4, which shows the total weighting function for $P-S$ backscattering at an image point located at increasing depth, in the center of a $500 \mathrm{~km}$-long array. The appearance of the weighting function is shown for various sampling densities (continuous, $5 \mathrm{~km}$ and $20 \mathrm{~km}$ station spacing). This test suggests that for an average global model (e.g., IASP91, Kennett and Engdahl [1991]) and a low-pass frequency cutoff of $0.3 \mathrm{~Hz}$, operator aliasing should affect the resulting image between the surface and a depth approximately equivalent to twice the station spacing. Caution should thus be taken when interpreting images in this depth range. Alternatively, it is possible to mitigate the effects of aliasing by muting the first 1-2 seconds of the scattered wavefield, as proposed by Poppeliers and Pavlis [2003ab]. However, this approach prevents the imaging of structure in the model's upper $\sim 10-20 \mathrm{~km}$.

\subsection{Model Space Assumptions}

In addition to the direct effects from source and station distribution, the resolution afforded by 2D GRT inversion may also be affected by departures from assumptions adopted in its theoretical development. In this section, we briefly discuss the implications of three such assumptions, namely the Born approximation, isotropy, and 2D geometry.

The Born approximation permits the linearization of the forward problem through the assumption that all wave propagation occurs through the unperturbed reference medium. This assumption is valid while the scattered component of the total wavefield remains much smaller than the unper- turbed wavefield. Detailed criteria for the validity of the Born approximation in seismic scattering can be found in Hudson and Heritage [1981]. Generally, material property perturbations should remain small (i.e., $\Delta \alpha \ll \alpha_{0}, \Delta \beta \ll \beta_{0}$, $\Delta \rho \ll \rho_{0}$ ) and the scale length of the scattering bodies should not exceed the dominant wavelength of the signal. Violation of these assumptions results in two main complications. First, it allows for significant multiple scattering to occur, which may result in the inclusion of artificial structure in the final image. Second, it modifies the predicted ray kinematics of the wavefield and thereby produces an incomplete focusing of scattered signal.

The 2D GRT inversion, as cast by Bostock et al.[2001], assumes that the scatterers and background reference model display isotropic elastic properties. This is a convenient and commonly employed assumption, as it reduces the number of independent inversion parameters to three unknowns (e.g., $\Delta \alpha / \alpha_{0}, \Delta \beta / \beta_{0}$, and $\left.\Delta \rho / \rho_{0}\right)$. However, as extensively reported in the literature [see Fischer et al., this volume], many types of mineral assemblages and structural features characterizing the crust and underlying mantle display strong coherent fabric that result in directional variations of elastic properties (i.e., anisotropy). The presence of anisotropy, if unaccounted for, may affect 2D GRT inversion images in two main ways: (1) structures will be mismapped and defocused due to altered ray kinematics [see, e.g. Larner and Cohen, 1993; Tsvankin, 1996]; (2) recovery of material property perturbations will be compromised due to the computation of incorrect radiation functions for the scattered waves [see Burridge et al., 1998]. A better characterization of anisotropic scattering parameters requires significant modifications to the current methodology, as discussed in section 5.

Lastly, the main reason for adopting a 2D formulation of the scattering problem is the limitation in currently available instrumentation, which precludes the deployment of seismometers over very dense grids necessary for a general 3D approach (see section 5 for a discussion on this matter). The 2D treatment may often be an oversimplifying assumption, yet it has been successfully used for many years in the oil industry [see, e.g., Yilmaz, 1987] and in crustal studies [e.g., Clowes et al., 1992]. Departures from this assumption may result in the inclusion of coherent signal from out-of-plane plane 3D scatterers in the final image. This signal will not necessarily map constructively but it will increase the level of background noise. It is thus important to test the validity of our approach by computing the minimum out-of-plane extent of 2D structure required to satisfy the assumption, and by comparing this value with information from regional tectonic models. Figure 5 presents a set of minimum values calculated for a global average model (i.e., iasp91) as a 

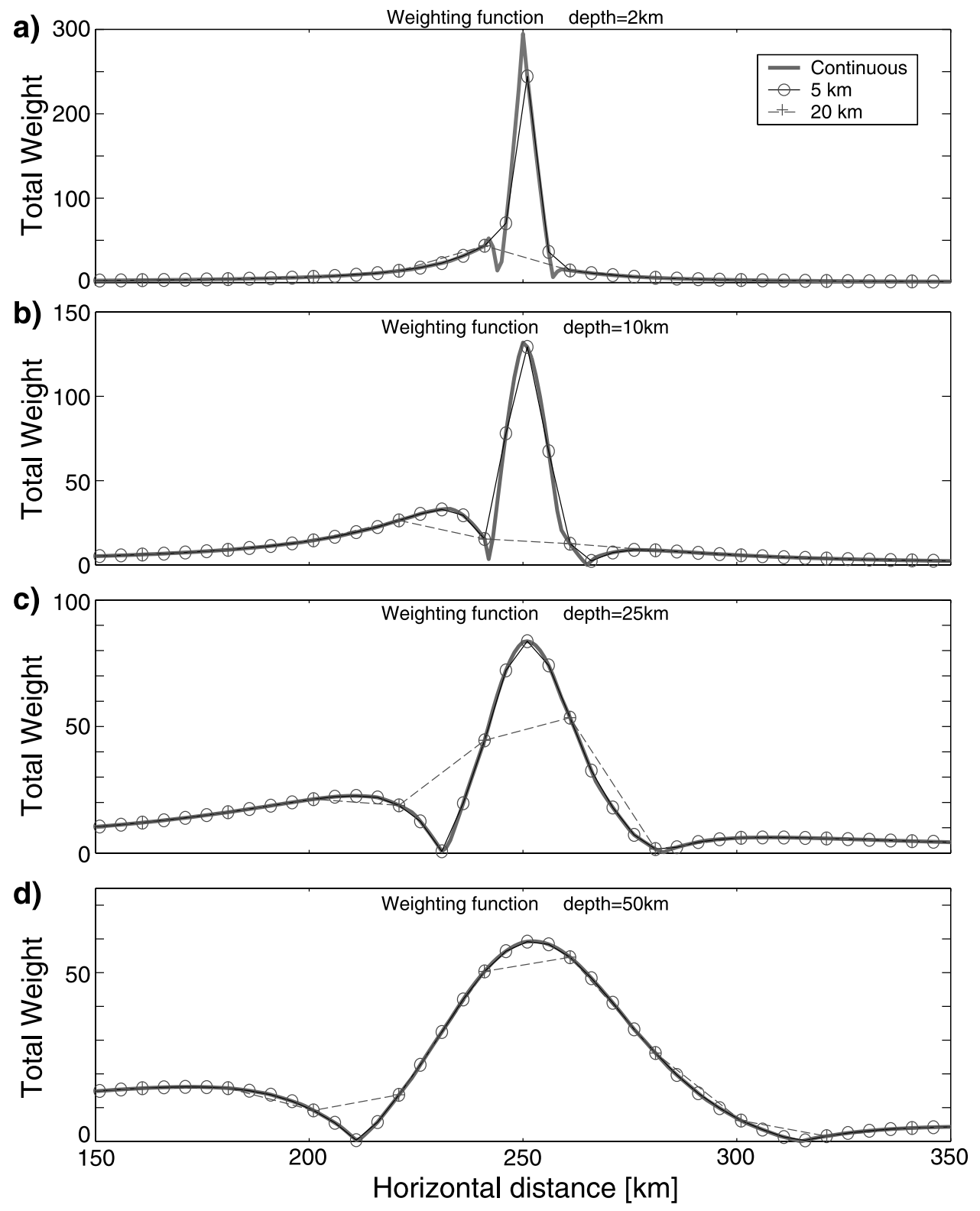

Figure 4. Sampling of the weighting function applied to the diffraction stacks (i.e., $\frac{1}{4 \pi}\left|\frac{\partial(\psi, \theta)}{\partial\left(x_{1}^{\prime}, \gamma\right)}\right| W_{l}(\theta) Y\left(\mathbf{x}_{0}, \mathbf{x}^{\prime}, \mathbf{p}_{\perp}^{0}\right)$ projected onto $\boldsymbol{\Delta} \boldsymbol{u}\left(\mathbf{x}^{\prime}, \mathbf{p}_{\perp}^{0}, t\right)$ in equation 7), for a single image point located at (a) $2 \mathrm{~km}$, (b) $10 \mathrm{~km}$, (c) $25 \mathrm{~km}$, and (d) $50 \mathrm{~km}$ beneath the center of a 500km-long array. The thick gray line represents continuous sampling, whereas solid and dashed black lines represent sampling at $5 \mathrm{~km}$ and $20 \mathrm{~km}$, respectively. Undersampling is manifest to depths equivalent to approximately $2 \times$ [station spacing]. 


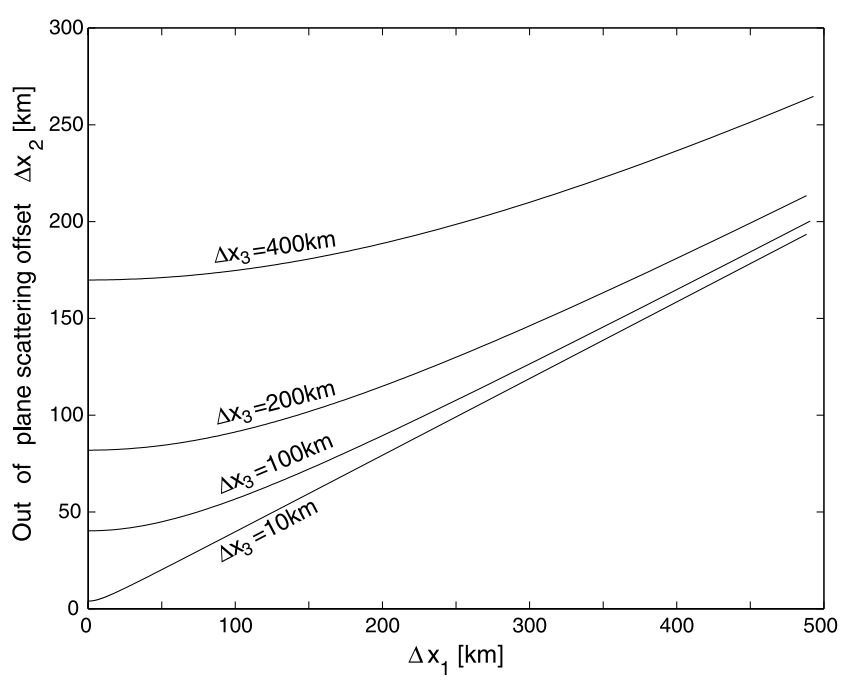

Figure 5. Minimum out-of-plane extent of 2D scatterer required by model-space assumptions, as a function of scatterer's depth $\left(\Delta x_{3}\right)$ and horizontal in-plane distance from recorder $\left(\Delta x_{1}\right)$.

function of scatterer's depth and horizontal in-plane distance from the recorder.

\section{APPLICATIONS TO FIELD DATA}

In this section, we apply 2D GRT inversion to data from two IRIS-PASSCAL experiments, ABI96 and LARSE. The main objective of this exercise is to assess the applicability of the method to deployments that do not meet its strict geometrical or data requirements. In the case of ABI96, station coverage is relatively sparse $(\sim 20 \mathrm{~km})$ and the data exhibit significant contamination by coherent noise and unmodelled teleseismic arrivals. For the LARSE array, the data are more densely sampled $(\sim 2 \mathrm{~km})$ but were recorded by short-period instruments, limiting the bandwidth of the recovered scattered signal. For an assessment of the full potential of 2D GRT inversion, the reader is referred to a previous application of the method to field data from the CASC93 experiment [see Rondenay et al., 2001; and Bostock et al., 2002].

\subsection{ABI96 Field Data}

During the summer of 1996, a portable array comprising 28 broadband three-component seismometers was deployed across the SE Canadian Shield, with an average station spacing of $20 \mathrm{~km}$. The array followed the main axis of the Lithoprobe Abitibi-Grenville transect [Ludden and Hynes, 2000], sampling Archean terranes of the Superior Province to the NW and Proterozoic rocks of the Grenville Province to the SE (Plate 1a). Results from a receiver function profile along this array were reported in Rondenay et al. [2000], and are reproduced here in Plate 1e. The colour scale represents the amplitude of the normalized impulse responses, with red and blue pulses denoting discontinuities with positive and negative downward velocity gradients, respectively. Starting from the southern end of the profile, Moho topography is apparent with northwestward crustal thickening from 40 to $45 \mathrm{~km}$, followed by an abrupt thinning of $\sim 10 \mathrm{~km}$ occurring some $65 \mathrm{~km}$ south of the Grenville Front (GF). This jump was interpreted as the signature of a suture zone complex, with the GF representing the surface expression of an associated crustal ramp. The presence of relict subducted crust in the Superior cratonic lithosphere is suggested by a sequence of negative polarity arrivals projecting northward into the mantle from the Moho step.

Two important issues regarding preprocessing must be considered before applying 2D GRT inversion to this data set. The first is data selection. Plate $1 \mathrm{~b}$ shows the locations of the 49 events selected to generate the 568 receiver functions used in the study of Rondenay et al. [2000]. The authors employed a simultaneous, frequency domain deconvolution algorithm [Gurrola et al., 1995], followed by mapping of time to depth. This process is loosely equivalent to a $1 \mathrm{D}$ post-stack migration operator and tends to enhance coherent signal related to continuous, sub-horizontal discontinuities relative to locally scattered signal or noise. In comparison, signal-to-noise ratio requirements for 2D GRT inversion are more stringent as this is a form of pre-stack migration that tends to carry the noise constructively into the final image. Due to this limitation, only 7 of the original 49 events were retained for the inversion (see Plate 1b). Poppeliers and Pavlis [2003b] proposed an approach to overcome this limitation by considering all major events recorded by an array and weighting them objectively based on the signal-to-noise ratio of their corresponding records.

The second issue regarding preparation of the ABI96 data is a recurrent problem of $P$-coda contamination by later arriving body-waves. Specifically, 3 of the 7 selected events displayed $P c P$ phases with significant amplitudes and pronounced moveout from $P$, which they trail by 30-100 s. This time difference results in the introduction of unwanted coherent $P c P$ signal in that portion of the $P$-coda which is sampled for scattered energy in the top $250 \mathrm{~km}$ of the model, thereby causing artificial structure in the resulting image. The same problem arises with teleseismic PP when attempting to image sublithospheric structure that require extended $(\sim 300 \mathrm{~s})$ data sections. In order to resolve this contamination problem, a series of preprocessing steps in addition to those in section 2.2 are implemented. These steps are 
a)

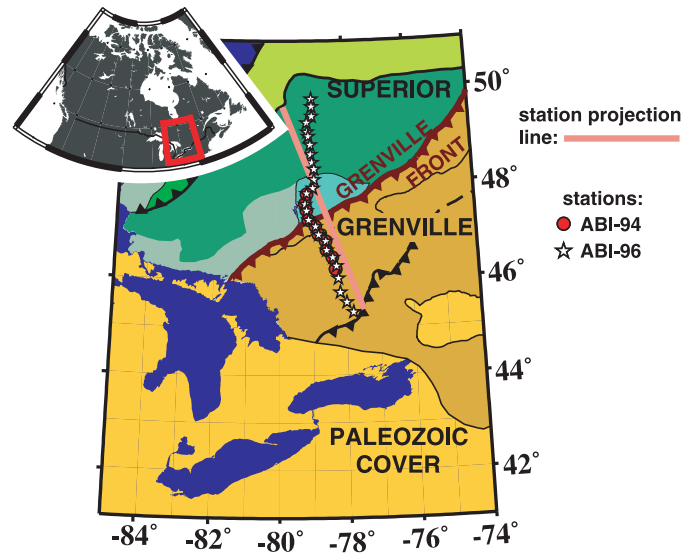

c)

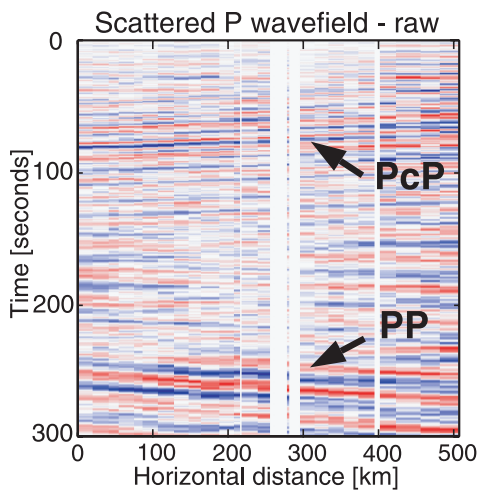

b)

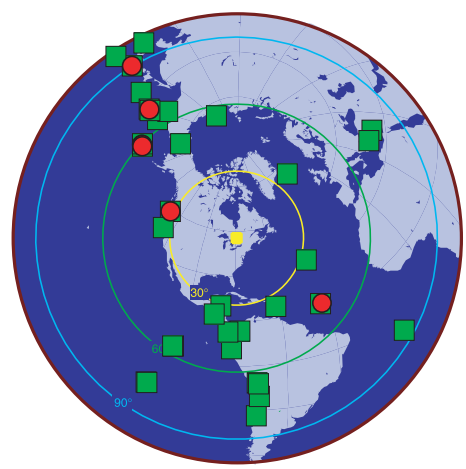

d)

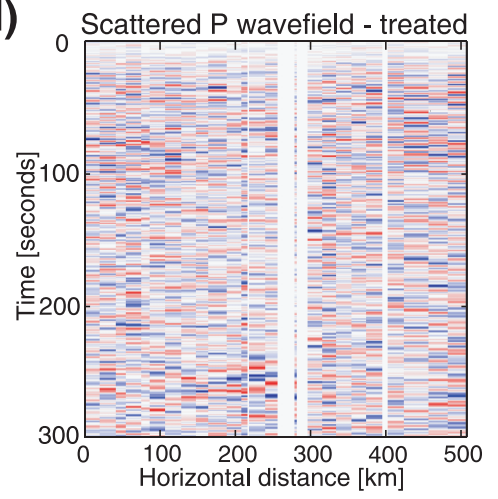

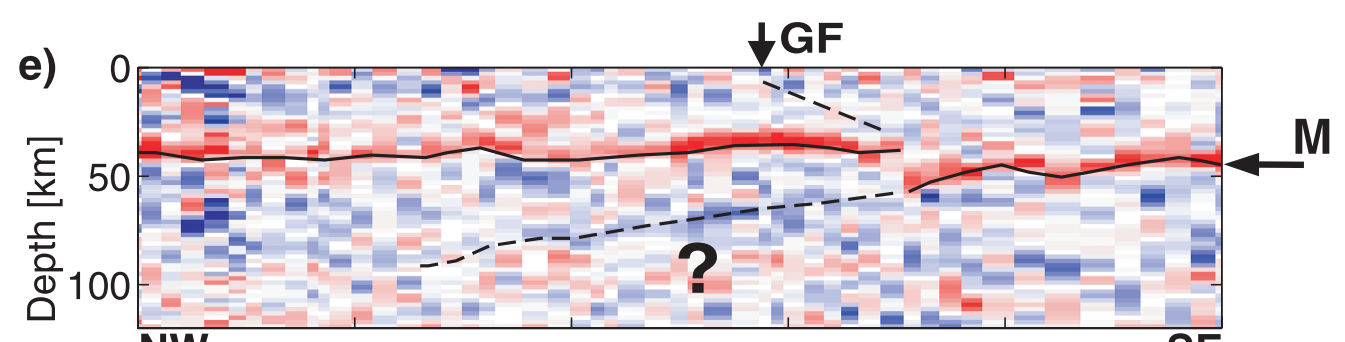

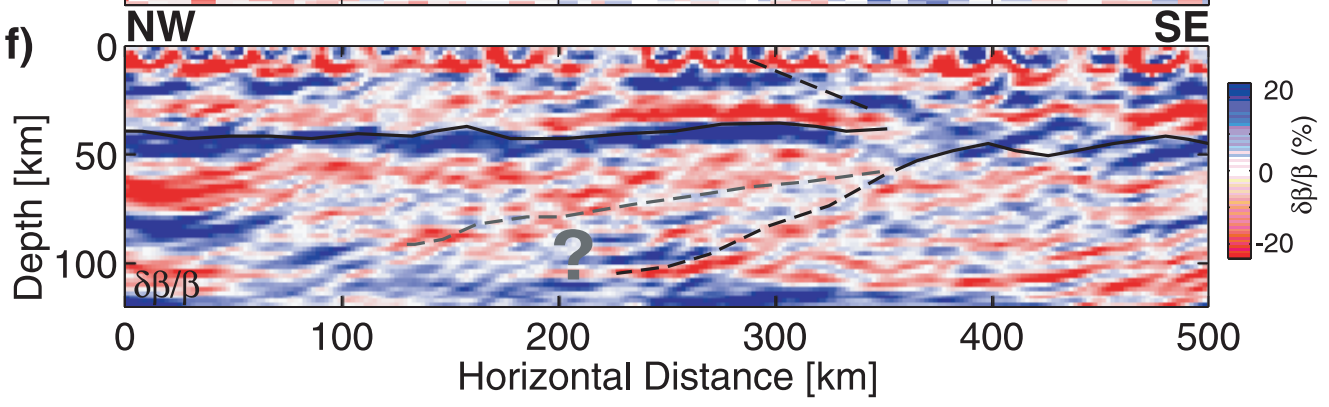

Plate 1. ABI96 experiment. a) Map of the study area. b) Distribution of events selected for receiver function analysis (green squares) and 2D GRT inversion (red circles). c) Scattered $P$-wavefield section obtain through preprocessing steps described in section 2.2. Note the contamination by teleseismic $P c P$ and $P P$ waves. d) Decontaminated $P$-wavefield section. e) Receiver function profile. Red and blue pulses denote discontinuities with positive and negative downward velocity gradients, respectively. Black lines show interpretation. Abbreviations: GF = Grenville Front, $\mathrm{M}=\mathrm{Moho}$. f) $S$ velocity perturbation profile obtained by $2 \mathrm{D}$ GRT inversion, with red to blue colour scale representing negative (slower) to positive (faster) velocity perturbations. 
applied prior to deconvolution of the scattered wavefield and can be summarized as follows:

(1) the traveltime and horizontal slowness of the corrupting phase are identified;

(2) the waveform of the corrupting phase is isolated using the inverse free surface transfer matrix;

(3) traces are optimally aligned with respect to this phase using multichannel cross-correlation;

(4) a principal component decomposition is applied and the first principal component, which contains the corrupting phase, is removed from the section;

(5) a clean data set is recovered by realigning the traces with respect to $P$ and multiplying by the free-surface transfer matrix.

This preprocessing sequence removes most of the unwanted signal, as illustrated in the example of Plate 1c-d. It is important to note, however, that it will also remove coherent scattered energy that displays the same moveout and polarization as the unwanted phase.

The 2D GRT inversion was applied to the preprocessed and decontaminated records from the seven selected events. Plate 1f shows the resulting $S$-velocity perturbation profile, with red to blue colour scale representing negative (slower) to positive (faster) velocity perturbations. In this type of profile, velocity discontinuities are thus denoted by colour contrasts (i.e., red-to-blue or blue-to-red). We note that the quality of the final image is remarkably high given the limited number of events retained for analysis. The Moho appears as a strong discontinuity that closely follows topographical variations observed on the RF profile (Plate 1e). It is important to note that whereas the RF image does not exploit free-surface multiples, the 2D GRT inversion relies primarily on these backscattered signals [see Shragge et al., 2001; Rondenay et al., 2001]. Consequently, there is a large degree of independence between the two images. The 2D GRT inversion also images a complex structure near the previously imaged Moho step. However, the feature in Plate 1f appears as less of a step and more as a series of imbricated ramps. Given the limited source/station distribution, it is difficult to assess whether this complex structure is real or if it is an artifact due to reduced coverage in $\nabla \mathcal{T}$ in the SE portion of the array.

We further note that 2D GRT inversion detects a possible discontinuity projecting into the mantle from the Moho disruption. This structure is better defined and possesses a greater dip $\left(\sim 22^{\circ}\right)$ than that observed in the RF profile. The dip is well within the range of dip resolution of $\left[-55^{\circ}, 60^{\circ}\right]$, which is afforded by the complete ABI96 dataset in this portion of the model (i.e., $x_{1}=300 \mathrm{~km}, x_{3}=80 \mathrm{~km}$ ). The mantle feature in Plate $1 \mathrm{f}$ is expected to be more robust, as 2D GRT inversion is more effective at constructively summing scattered signal emanating from dipping interfaces. However, greater source coverage would be required to confirm this structure.

The resolution of the final image (Plate 1f) is affected by two of the factors discussed in section 3. First, the large station spacing places the top portion of the model in the region affected by stacking weight aliasing. According to the rule of thumb devised in section 3.2, the affected region should extend between $0-40 \mathrm{~km}$ depth (i.e., twice the station spacing). In the present case, aliasing results in a high wavenumber "speckle" which distorts the image mostly between $0-20 \mathrm{~km}$ depth. This implies that the predicted extent of the affected region can be reduced, in part, by the simultaneous treatment of multiple sources. Second, due to the incomplete and irregular coverage in $\nabla \mathcal{T}$ at each image point (see Figure $3 \mathrm{~d}$ ), the magnitudes of material property perturbation (i.e., $\delta \beta / \beta_{0}$ in Plate 1f) are not correctly recovered. Therefore, when dealing with limited data sets, the resulting profiles should not be interpreted quantitatively.

In summary, this application of 2D GRT inversion demonstrates that while sparse data sampled at $\sim 20 \mathrm{~km}$ intervals may not be ideal, they nonetheless permit meaningful imaging of structure in the lower crust and underlying lithospheric mantle.

\subsection{LARSE Field Data}

During the fall of 1993, a linear array of 89 vertical and three-component short-period seismometers was deployed across the Los Angeles Basin, San Gabriel Mountains and Mojave Desert, as part of the LARSE experiment [Plate 2a; see Kohler and Davis, 1997]. The array was $175 \mathrm{~km}$ long with a $\sim 2 \mathrm{~km}$ station spacing, and it was designed to perpendicularly intersect the dominantly $2 \mathrm{D}$ regional structure, in particular the San Andreas Fault. A receiver function profile beneath this array was produced by Zhu [2000] using 941 traces (from 30 events) binned according to Common Conversion Points. This study identified abrupt vertical offsets in Moho depth occurring directly beneath the surface location of the San Andreas Fault and the Eastern California Shear Zone, supporting a thick-skinned behaviour for these two crustal structures.

We introduce this data set to demonstrate two important issues in imaging lithospheric structure using teleseismic body-wave data, namely the contaminating influences of unmodelled scattering and errors in the estimated source time function. In Plate $2 \mathrm{c}$ we show the result of preprocessing only vertical component seismograms to produce an estimate of the scattered wavefield. Partly coherent signals with hy- 
a)

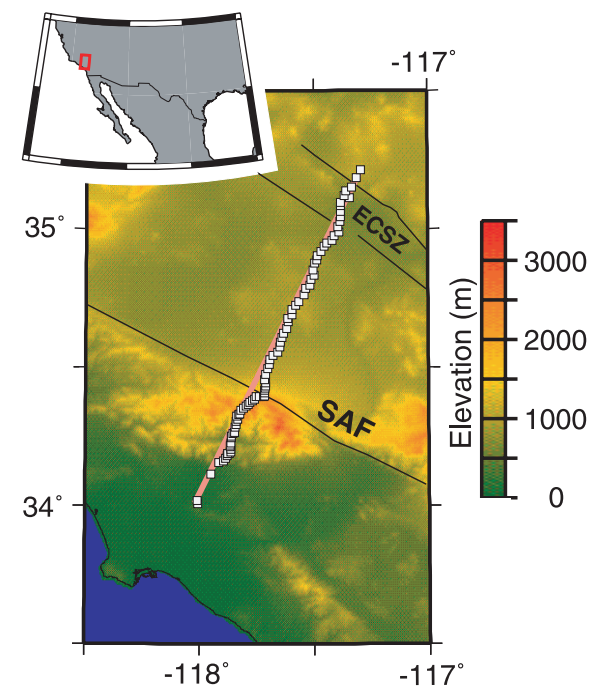

c)

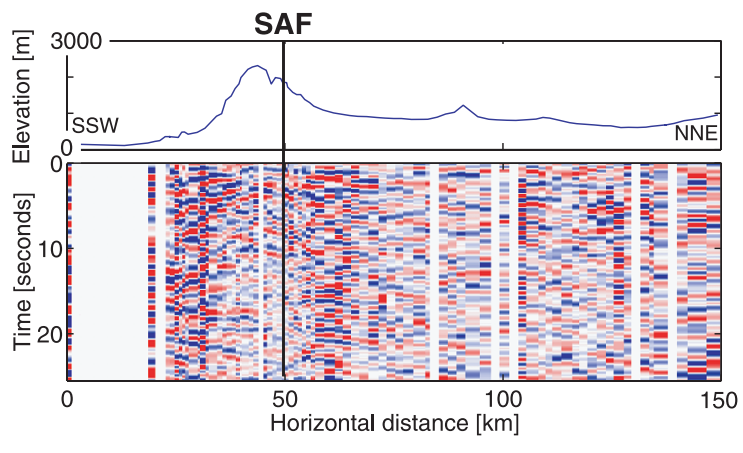

b)
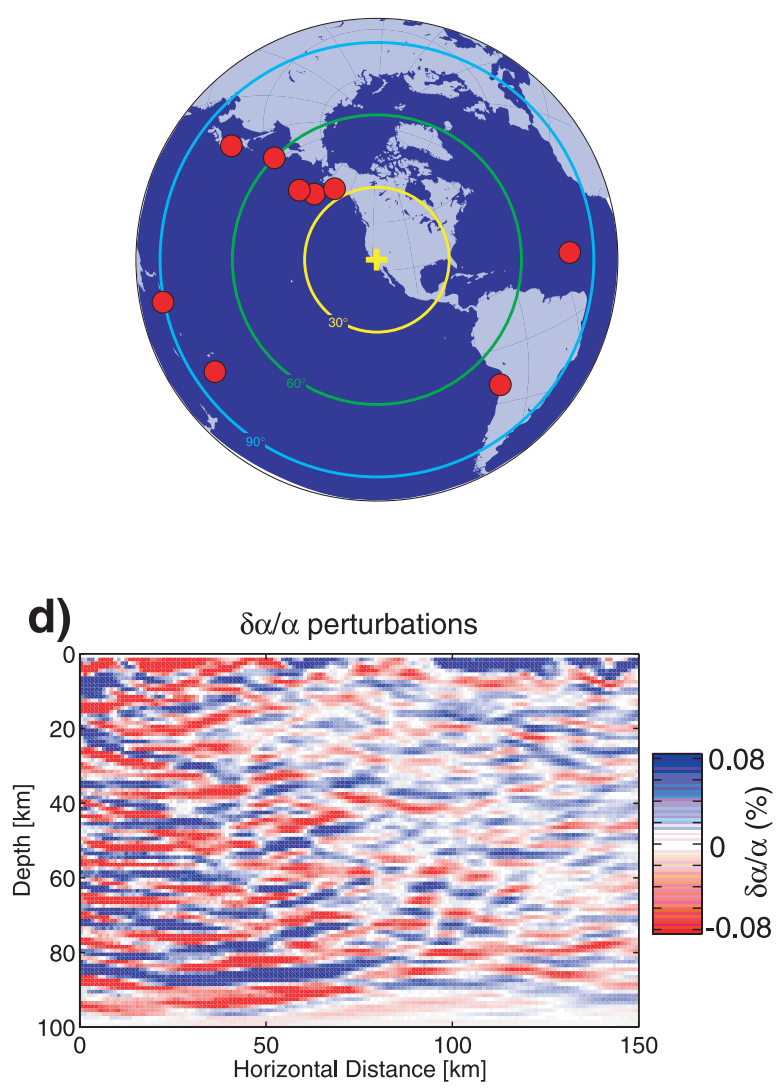

Plate 2. LARSE experiment. a) Map of the study area. Abbreviations: SAF = San Andreas Fault, ECSZ = Eastern California Shear Zone. b) Distribution of events selected for 2D GRT inversion. c) Top panel: surface topography along the profile. Lower panel: scattered $P$-wavefield section for a Kamchatka earthquake (Nov. 17, 1993; 11:18:50 UT; $\left.51.79^{\circ} \mathrm{N}, 158.67^{\circ} \mathrm{E}, 24 \mathrm{~km} ; m_{\mathrm{b}}=6.1\right)$. Note the presence of coherent diffractions hyperbolas centered near distance $43 \mathrm{~km}$, coinciding closely with maximum in topography. d) $P$-velocity perturbation profile obtained by $2 \mathrm{D}$ GRT inversion, with colour scale defined as for Plate 1f. 
perbolic moveout are evident and centered near distance $43 \mathrm{~km}$ along the profile. At first glance this observation is encouraging as it suggests the presence of strong localized subsurface heterogeneity. However, closer examination indicates that the apparent velocity of these signals is approximately $3.2 \mathrm{~km} / \mathrm{s}$. As the true wave velocity can only be less than the apparent velocity, this energy most likely represents Rayleigh waves originating through scattering from off-axis, surface topography. This inference is supported by a plot of surface topography along the profile wherein it is noted that the hyperbola apices coincide closely with the maximum in topography and topographic gradient (Plate 2c). The particle motions of Rayleigh waves also project onto the horizontal components and it is therefore important to assess and minimize their effect on images of the immediately underlying San Andreas fault as produced using more traditional receiver function approaches [Zhu, 2000; see further discussion in section 5].

The image resulting from inversion of the short-period vertical component data from 9 events (see Plate $2 b$ ) are shown in Plate $2 \mathrm{~d}$. There is little in the image that can be confidently attributed to major lithospheric structure. In particular, the Moho, which is evident in images that incorporate horizontal component (i.e., $S$-wave) information [e.g., $Z h u, 2000]$, cannot be identified using free-surface $P$-wave multiples. The primary reason for this is that our source estimation procedure is ineffective in distinguishing signal generated by near-horizontal planar structure from complexity in the source time function. The resulting errors in the source-time function have a much larger effect on $P$-wave data than $S$-wave data because the $P$-wave data are dominated by the incident wave whereas $S$-wave data contain, by definition, only scattered wave energy.

In summary, this example demonstrates that there are several factors that compromise the use of 2D GRT inversion on short-period, vertical component data sets. Therefore, application of the method in its present form is not recommended for this category of experimental setup. Nevertheless, possible future improvements discussed in the next section may change this situation.

\section{FUTURE DIRECTIONS}

We now build on our understanding of the factors affecting resolution to discuss a series of improvements that may render the GRT inversion approach more robust and more generally applicable. Five main points are considered.

First, the GRT inversion could be readily implemented for 3D isotropic scattering. This would permit better focusing of signal scattered at realistic subsurface structure. The theory has already been developed for the acoustic [Miller et al., 1987] and elastic [Beylkin and Burridge, 1990] treatment of seismic reflection data. The only caveat to 3D GRT inversion lies in the considerable, and currently prohibitive requirements for recording instruments. For example, a grid of 225 recording nodes (equipped with broadband seismometers) would be necessary to image a region of $300 \mathrm{~km}$ $\times 300 \mathrm{~km}$ with an average station spacing of $20 \mathrm{~km}$. It is possible that the requirement of relatively even station spacing may be relaxed by applying the pseudostation stacking approach of Neal and Pavlis [1999], which allows the projection of sparse data sets onto a regular grid. This approach has been employed in teleseismic GRT imaging by Poppeliers and Pavlis [2003a-b].

Second, the GRT inversion can be made more general by considering the inverse scattering problem for an anisotropic, elastic medium. Such anisotropic approaches have already been implemented by Burridge et al. [1998] for seismic reflection data, and by Bostock [2002] and Bank and Bostock [2002] for the 1D teleseismic case. The main challenge with generalization to an anisotropic medium lies in the large number of independent parameters that are inverted for. There are 22 independent parameters in the most general case, comprising 21 elastic tensor components plus density. Note, however, that this number is reduced to $9+1$ parameters for orthorhombic systems (e.g., olivine) and to $5+1$ parameters for hexagonal symmetry. Given the limited source-receiver coverage, the problem is generally underdetermined and only certain linear combinations of anisotropic parameters can be recovered [Burridge et al., 1998]. Furthermore, it is not obvious that the data sampling and signal-to-noise levels afforded by teleseismic waves will generally be sufficient to contemplate fully 3D inversion for anisotropic structure. Nevertheless, this type of analysis would prove useful in estimating the fraction of the scattered signal that can only be explained by anisotropic perturbations.

Third, as we have noted from our analysis of the LARSE data set, unmodelled teleseismic body-to-surface wave scattering [Revenaugh, 1995b; Abers, 1998] poses a serious impediment to the utilization of higher frequency data in resolving fine scale structure. This form of scattering is most severe at short periods $(<2 s)$. From the observations we have made to date in subduction zones [e.g., Rondenay et al., 2001], there is apparently a mid-period band (3-10 s, accessible using broadband instrumentation) wherein scattering is largely coherent and relatively unaffected by topography. Extending the useful band to still higher frequencies may be possible by developing 3D generalizations of the 1D free-surface forward and inverse transfer operators [Kennett, 1991; Bostock and Rondenay, 1999a] employed in the present work. Using densely sampled topographic data bases, it 
may thereby be possible to attenuate surface wave signals in the preprocessing sequence.

Fourth, estimation of the source-time function is another area of research where improvements are needed if structural information from intra-mode (e.g., $P$-to- $P, S$-to- $S$ ) scattering is to be extracted. Our use of principal component analysis to attempt separation of incident and scattered wavefields fails to accurately separate low-frequency scattered signal from near-horizontal, planar structure. As a result, the Moho, for example, is generally not identifiable on images constructed from free-surface $P$-to- $P$ multiples. It is possible that this problem can be overcome by exploiting contrasting statistical properties of the source and scattering to better accomplish their separation.

Finally, it would be desirable to move beyond the Born or single-scattering approximation in teleseismic imaging. The effect of this assumption is manifest in two ways. Firstly, if the background medium does not accurately capture the long wavelength components of the true Earth's velocity, imaged structure will be distorted and mispositioned. Better estimates of large-scale velocity structure can be achieved through contemporaneous tomographic analysis of the traveltimes of direct waves. Alternatively, newly developed approaches [Weglein et al., 2002] that exploit higher order terms in the Born and inverse scattering series may be applicable in the teleseismic context. However, these approaches rely heavily on an accurate knowledge of scattered wave amplitudes that may be difficult to achieve in practical circumstances.

A second consequence of the single-scattering approximation is the appearance of image artifacts related to discrete, multiply scattered arrivals. These include most notably freesurface multiples. Our 2D GRT approach partially accounts for the free-surface multiples by considering them as generated by image sources located above the free-surface and incorporating them within the inversion. However, because we do not isolate each different multiple mode into separate data sections, they cross contaminate one another in the resulting images [Shragge et al., 2001]. The machinery afforded by the Born/inverse scattering series [Weglein et al., 1997] may provide a means of isolating individual data sections corresponding to each free-surface multiple as part of the preprocessing sequence. The individual data sections can then be inverted simultaneously to produce images which do not possess cross-mode contamination. This approach is currently a topic of investigation.

\section{CONCLUSIONS}

Until now, the 2D GRT inversion presented in Bostock et al., [2001] has only been applied to ideal synthetic and field data that are not necessarily representative of typical experimental setups. In this paper, we have assessed the resolving power of the approach for variable field conditions. In particular, we demonstrated that: (1) comprehensive source/station coverage is necessary to optimize geometrical resolution and recover accurate material property perturbations; (2) the range in dip resolution diminishes with increasing depth and is inversely proportional to array width (e.g., reaches $\left[-45^{\circ}, 45^{\circ}\right]$ at depths equivalent to $\sim 1 / 2$ array width); (3) distortion of the image due to spatial aliasing is only significant at depths $\leq 2 \times$ [station spacing]; and (4) unaccounted for departures from model assumptions (i.e., isotropy and 2D geometry) result in defocusing and mismapping of structure.

We have further illustrated the potential and limitations of 2D GRT inversion through two new applications to field data. Application to ABI96 required the introduction of additional preprocessing steps to minimize contamination of the data by $P c P$ and $P P$ waves. Results demonstrated that structure from the lower crust downward could be successfully imaged by broadband data from relatively sparse seismic arrays with $\sim 20 \mathrm{~km}$ station spacing. Limited source distribution was shown to hinder the recovery of accurate material property perturbations, but the resulting images could still be interpreted qualitatively. Application to LARSE showed that images obtained from high frequency records were subject to significant contamination by scattered surface waves. Further deterioration of resolution was attributed to a loss of scattered signal through the preprocessing steps currently used to render the data amenable to analysis. The development of improved preprocessing tools that could isolate the different scattering modes from the incident wavefield and corrupting phases is the focus of ongoing research.

Acknowledgments. We thank Biondo Biondi, Gary Pavlis and Alan Levander for their constructive reviews. The data used in this paper were obtained through the IRIS Data Management Center, which is funded through NSF grant EAR-0004370. The ABI96 experiment was co-funded by IRIS-PASSCAL and LITHOPROBE, and the LARSE experiment was funded by IRIS-PASSCAL. We acknowledge all the people involved in these experiments, and thank in particular Monica Kohler for providing extra information on the LARSE experiment. The authors made use of the Generic Mapping Tool [Wessel and Smith, 1995] for some figures. This work was supported by a Natural Science and Engineering Research Council of Canada (NSERC) postdoctoral fellowship to SR.

\section{REFERENCES}

Abers, G., Array measurements of phase used in receiver-functions calculations: importance of scattering, Bull. Seismol. Soc. Am., 88, 313-318, 1998. 
Aki, K., A. Christofferson, and E. S. Husebye, Determination of the three-dimensional seismic structure of the lithosphere, $J$. Geophys. Res., 82, 277-296, 1977.

Bank, C.-G., and M. G. Bostock, Linearized inverse scattering of teleseismic waves for anisotropic crust and upper mantle structure: 2. Numerical examples and applications to data from Canadian stations, J. Geophys. Res. 108(B5), 2259, doi:10.1029/ 2002JB001951, 2003.

Beylkin, G., Imaging of discontinuities in the inverse scattering problem by inversion of a causal generalized Radon transform, J. Math. Phys., 26, 99-108, 1985.

Beylkin, G., and R. Burridge, Linearized inverse scattering problem by inversion of a causal generalized Radon transform, Wave Motion, 12, 15-52, 1990.

Bostock, M. G., Kirchhoff-approximate inversion of teleseismic wavefields, Geophys. J. Int., 149, 787-795, 2002.

Bostock, M. G., Linearized inverse scattering of teleseismic waves for anisotropic crust and mantle structure: 1. Theory, J. Geophys. Res., 108(B5), 2258, doi:10.1029/2002JB001950, 2003.

Bostock, M. G., and S. Rondenay, Migration of scattered teleseismic body waves, Geophys. J. Int., 137, 732-746, 1999a.

Bostock, M. G., and S. Rondenay, Corrigendum to "Migration of scattered teleseismic body waves", Geophys. J. Int., 139, 597, 1999b.

Bostock, M. G., S. Rondenay, and J. Shragge, Multiparameter twodimensional inversion of scattered teleseismic body waves, 1 , Theory for oblique incidence, J. Geophys. Res., 106, 30,77130,782, 2001.

Bostock, M. G., R. D. Hyndman, S. Rondenay, and S. M. Peacock, An inverted continental Moho and serpentinization of the forearc mantle, Nature, 417, 536-538, 2002.

Burridge, R., M. V. de Hoop, D. Miller, and C. Spencer, Multiparameter inversion in anisotropic elastic media, Geophys. J. Int., 134, 757-777, 1998.

Clowes, R. M., F. A. Cook, A. G. Green, C. E. Keen, J. N. Ludden, J. A. Percival, G. M. Quinlan, and G. F. West, Lithoprobe: new perspectives on crustal evolution, Can. J. Earth Sci., 29, 18131864, 1992.

Dueker, K. G., and A. F. Sheehan, Mantle discontinuity structure from midpoint stacks of converted $P$ to $S$ waves across the Yellowstone hotspot track, J. Geophys. Res., 102, 8313-8327, 1997.

Frederiksen, A. W., and J. Revenaugh, Lithospheric imaging via teleseismic scattering tomography, Geophys. J. Int., submitted, 2002.

Grand, S. P., Mantle shear structure beneath the Americas and surrounding oceans, J. Geophys. Res., 99, 11591-11621, 1994.

Gurrola, H., G. E. Baker, and J. B. Minster, Simultaneous timedomain deconvolution with application to the computation of receiver functions, Geophys. J. Int., 120, 537-543, 1995.

Hudson, J. A., and J. R. Heritage, The use of the Born approximation in seismic scattering problems, Geophys. J. Roy. Astr. S., 66, 221-240, 1981.

Kennett, B. L. N., The removal of free surface interactions from three-component seismograms, Geophys. J. Int., 104, 153-163, 1991.

Kennett, B. L. N., and E. R. Engdahl, Travel times for global earthquake location and phase identification, Geophys. J. Int., 105, 429-465, 1991.

Kohler, M. D., and P. M. Davis, Crustal thickness variations in Southern California from Los Angeles Region Seismic Experiment passive phase teleseismic travel times, Bull. Seis. Soc. Am., 87, 1330-1344, 1997.

Langston, C. A., Structure under Mount Rainier, Washington, inferred from teleseismic body waves, J. Geophys. Res., 84, 47494762, 1979.

Larner, K. L., and J. K. Cohen, Migration error in transversely isotropic media with linear velocity variation with depth, Geophysics, 58, 1454-1467, 1993.

Levander, A., E. Humphreys, G. Ekstrom, A. Meltzer, and P. Shearer, Proposed project would give unprecedented look under North America, Eos Trans. AGU, 80, 245, 1999.

Li, A., D. W. Forsyth, and K. M. Fischer, Shear velocity structure and azimuthal anisotropy beneath eastern North America from Rayleigh wave inversion, J. Geophys. Res. 108(B8), 2362, doi:10.1029/2002JB002259, 2003.

Ludden, J., and A. Hynes, The Lithoprobe Abitibi-Grenville transect: two billion years of crust formation ad recycling in the Precambrian Shield of Canada, Can. J. Earth Sci., 37, 459476, 2000.

Miller, D., M. Oristaglio, and G. Beylkin, A new slant on seismic imaging: Migration and integral geometry, Geophysics, 52, 943964, 1987.

Poppeliers C., and G. L. Pavlis, Three-dimensional, prestack, plane wave migration of teleseismic $P$-to- $S$ converted phases: 1. Theory, J. Geophys. Res., 108 (B2), 2112, doi:10.1029/ 2001JB000216, 2003a.

Poppeliers, C. and G. L. Pavlis, Three-dimensional, prestack, plane wave migration of teleseismic $P$-to- $S$ converted phases: 2 . Stacking multiple events, J. Geophys. Res., 108(B5), 2267, doi:10.1029/2001JB001583,2003b.

Revenaugh, J., A scattered-wave image of subduction beneath the Transverse Ranges, Science, 268, 1888-1892, 1995 a.

Revenaugh, J., The contribution of topographic scattering to teleseismic coda in Southern California, Geophys. Res. Lett., 22, 543-546, 1995 b.

Rondenay, S., M. G. Bostock, T. M. Hearn, D. J. White and R. M. Ellis, Lithospheric assembly and modification of the SE Canadian Shield: Abitibi-Grenville Teleseismic Experiment, $J$. Geophys. Res., 105, 13,735-13,754, 2000.

Rondenay, S., M. G. Bostock, and J. Shragge, Multiparameter twodimensional inversion of scattered teleseismic body waves, 3, Application to the Cascadia 1993 data set, J. Geophys. Res., 106, 30,795-30,808, 2001.

Ryberg, T., and M. Weber, Receiver function arrays: a reflection seismic approach, Geophys. J. Int., 141, 1-11, 2000.

Sheehan, A. F., P. M. Shearer, H. J. Gilbert, and K. G. Dueker, Seismic migration processing of $P-S V$ converted phases for man- 
tle discontinuity structure beneath the Snake River Plain, western United States, J. Geophys. Res., 105, 19055-19065, 2000.

Shragge, J., M. G. Bostock, and S. Rondenay, Multiparameter twodimensional inversion of scattered teleseismic body waves, 2, Numerical examples, J. Geophys. Res., 106, 30,783-30,794, 2001.

Tsvankin, I., $P$-wave signatures and notation for transversely isotropic media: An overview, Geophysics, 61, 467-483, 1996.

Ulrych, T. J., M. D. Sacchi, and S. L. M. Freire, Eigenimage processing of seismic sections, in Covariance Analysis of Seismic Signal Processing, edited by R. L. Kirlin, and W. J. Done, SEG Monograph, Tulsa, 1998.

VanDecar, J. C., Upper-mantle structure of the Cascadia subduction zone from non-linear teleseismic travel-time inversion, Ph.D. thesis, 165 pp., Univ. of Wash., Seattle, June 1991.

VanDecar, J. C., and R. S. Crosson, Determination of teleseismic relative phase arrival times using multi-channel cross-correlation and least squares, Bull. Seismol. Soc. Am., 80, 150-159, 1990.

van der Hilst, R. D., S. Widiyantoro, and E. R. Engdahl, Evidence for deep mantle circulation from global tomography, Nature, 386, 578-584, 1997

van der Lee, S., and G. Nolet, Upper mantle $S$ velocity structure of North America, J. Geophys. Res., 102, 22,815-22,838, 1997.

Vinnik, L. P., Detection of waves converted from $P$ to $S V$ in the mantle, Phys. Earth Planet. Inter., 15, 39-45, 1977.
Weglein, A. B., F. A. Gasparatto, P. M. Carvalho, and R. H. Stolt, An inverse-scattering series method for attenuating multiples in seismic reflection data, Geophysics, 62, 1975-1989, 1997.

Weglein, A. B., D. J. Foster, K. H. Matson, S. A. Shaw, P. M. Carvalho, and D. Corrigan, Predicting the correct spatial location of reflectors without knowing or determining the precise medium and wave velocity: Initial concept, algorithm and analytic and numerical example, J. Seism. Explor., 10, 367-382, 2002.

Wessel, P., and W. H. F. Smith, New version of the Generic Mapping Tools released, Eos Trans. AGU, 76, 329, 1995.

Yilmaz, O., Seismic data processing, Society of Exploration Geophysicists, Tulsa, OK, 1987.

Zhu, L., Crustal structure across the San Andreas Fault, southern California from teleseismic converted waves, Earth Planet. Sc. Lett., 179, 183-190, 2000.

S. Rondenay, Department of Earth, Atmospheric and Planetary Sciences, Massachusetts Institute of Technology, 77 Massachusetts Ave, Cambridge, MA 02129. (e-mail:rondenay@mit.edu)

M.G. Bostock, Department of Earth and Ocean Sciences, University of British Columbia, 2219 Main Mall, Vancouver, British Columbia, Canada, V6T 1Z4. (e-mail:bostock@eos.ubc.ca

K.M. Fischer, Department of Geological Sciences, Brown University, P.O. Box 1846, Providence, RI 02912. (e-mail: Karen_Fischer@brown.edu) 